\title{
Rhetoric and Composition
}

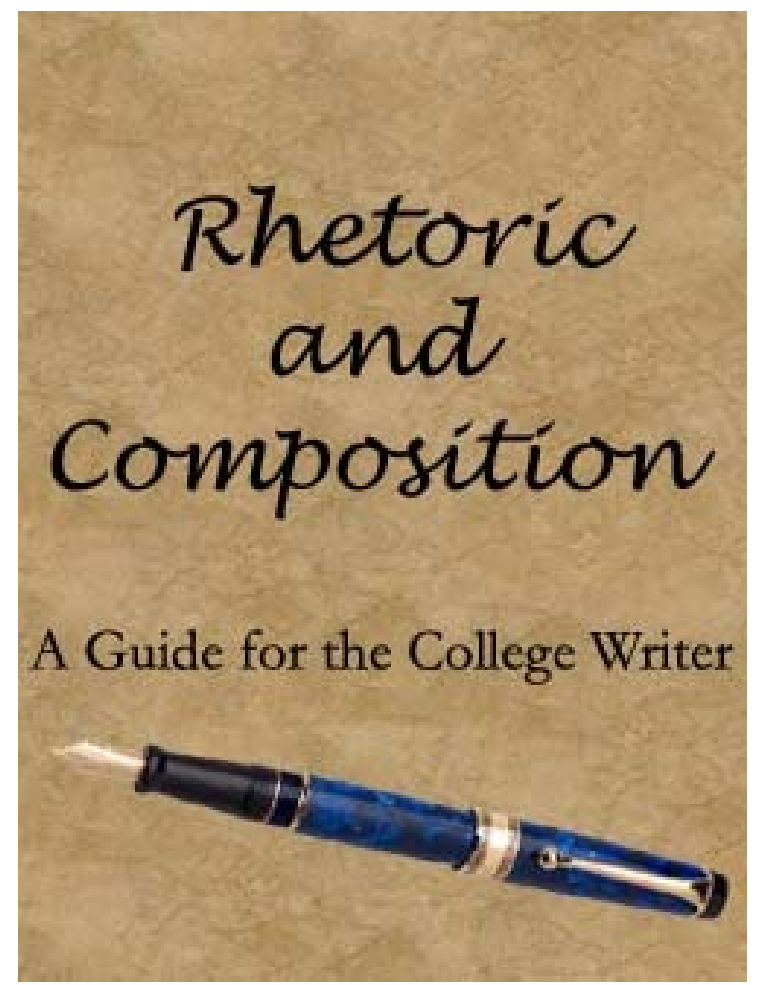

Rhetoric and Composition

Welcome to the Rhetoric and Composition Wikibook, designed for use as a textbook in first-year college composition programs, written as a practical guide for students struggling to bring their writing up to the level expected of them by their professors and instructors. For more detail, see the Introduction chapter.

\section{Contents}

1. Introduction

2. Overview: The Writing Process
(a) Planning and Prewriting
(b) Collaborating
(c) Researching
(d) Drafting
(e) Editing
(f) Reviewing
(g) Revising
(h) Proofreading

\author{
(i) Publishing
}

3. Genres
(a) Memoirs
(b) Reviews
(c) Commentaries
(d) Proposals

4. Writing Applications
(a) Analyzing Assignments
(b) Description
(c) Narration
(d) Exposition
(e) Evaluation
(f) Argument

5. Advanced Topics
(a) Writing in the Humanities
(b) Writing in the Sciences
(c) Writing in Business
(d) Oral Presentations
(e) Rhetorical Analysis
(f) Annotated Bibliography
(g) Writing for the Web
i. Formality
ii. Formatting
iii. Utility
iv. Navigation
v. Establishing Connections
vi. Advice for Writing in Various Venues on the Web
vii. Usability Testing
viii. Useful Links
ix. See Also

6. Grammar and Mechanics
(a) Parts of Speech
(b) Parts of the Sentence
(c) Types of Sentences
(d) Active and Passive Voice
(e) Punctuation
(f) Commas 

(g) Semicolons
(h) Colons
(i) Apostrophes
(j) Quotation Marks
(k) Hyphens and Dashes
(l) Parentheses
(m) Capitalization
(n) Common Errors
(o) Writing Feedback
(p) Citing Sources
(q) Plagiarism

7. Teacher's Handbook
(a) Teaching With Wikitexts
(b) Creating Effective Assignment Sequences
(c) Writing as a Process
(d) Modes of Writing
i. Description
ii. Narration
iii. Exposition
iv. Evaluation
v. Argumentation
(e) Annotated Bibliography
(f) Rhetorical Analysis
(g) Sentence Structure
(h) Grammar
(i) Writing in the Humanities
(j) Writing in the Sciences
(k) Writing in Business
(l) Oral Presentations
(m) Writing for the Web
(n) Using the Portfolio Method
(o) Assignment Sheet Database
(p) Handout Database
(q) Lesson Plan Database

8. Appendices
(a) Glossary
(b) Index
(c) Detailed TOC
(d) Bibliography
(e) Authors
(f) Ask a Question!
(g) Feature Requests

\section{Related books}

- English 


\section{Text and image sources, contributors, and licenses}

\subsection{Text}

- Rhetoric and Composition Source: https://en.wikibooks.org/wiki/Rhetoric_and_Composition?oldid=2464746 Contributors: Liblamb, DavidCary, Derbeth, Mattbarton.exe, Klingoncowboy4, Whiteknight, Jer54, Kellin, Oyvind enwikibooks, Jguk, Hagindaz, Az1568, Thenub314, Kubbs17, Xania, Tigerrat29, Jess0501, Herbythyme, Herraotic, Admax enwikibooks, Klka0701, B.Doberstein, Apickens, Mudbat, Mike's bot account, Joshboyd, Reywas92, Dan Polansky, Dallas1278, Melindakay, Sigma 7, Adrignola, Belteshazzar, ErinSchaefer and Anonymous: 26

\subsection{Images}

- File:100_percents.svg Source: https://upload.wikimedia.org/wikipedia/commons/c/c7/100_percents.svg License: CC0 Contributors: File: $100 \%$.svg Original artist: Siebrand

- File:25_percents.svg Source: https://upload.wikimedia.org/wikipedia/commons/c/ce/25_percent.svg License: CC0 Contributors: File: $25 \%$.svg Original artist: Ftiercel

- File:50_percents.svg Source: https://upload.wikimedia.org/wikipedia/commons/6/62/50_percents.svg License: CC0 Contributors: File: 50\%.svg Original artist: Ftiercel

- File:75_percents.svg Source: https://upload.wikimedia.org/wikipedia/commons/6/62/75_percent.svg License: CC0 Contributors: File: $75 \%$.svg Original artist: Ftiercel

- File:Commons-logo.svg Source: https://upload.wikimedia.org/wikipedia/commons/4/4a/Commons-logo.svg License: Public domain Contributors: This version created by Pumbaa, using a proper partial circle and SVG geometry features. (Former versions used to be slightly warped.) Original artist: SVG version was created by User:Grunt and cleaned up by 3247, based on the earlier PNG version, created by Reidab.

- File:Gnome-mime-application-pdf.svg Source: https://upload.wikimedia.org/wikipedia/commons/b/b6/ Gnome-mime-application-pdf.svg License: LGPL Contributors: http://ftp.gnome.org/pub/GNOME/sources/gnome-themes-extras/ 0.9/gnome-themes-extras-0.9.0.tar.gz Original artist: David Vignoni

- File:Printer.svg Source: https://upload.wikimedia.org/wikipedia/commons/2/23/Printer.svg License: Public domain Contributors: The Tango! Desktop Project Original artist: The people from the Tango! project

- File:Rc-cover.jpg Source: https://upload.wikimedia.org/wikibooks/en/0/05/Rc-cover.jpg License: GFDL Contributors: ? Original artist:

- File:Wikipedia-logo.svg Source: https://upload.wikimedia.org/wikipedia/commons/8/80/Wikipedia-logo-v2.svg License: CC BY-SA 3.0 Contributors: File:Wikipedia-logo.svg as of 2010-05-14T23:16:42 Original artist: version 1 by Nohat (concept by Paullusmagnus); Wikimedia.

- File:Wikiquote-logo.svg Source: https://upload.wikimedia.org/wikipedia/commons/f/fa/Wikiquote-logo.svg License: Public domain Contributors: Own work Original artist: Rei-artur

- File:Wikiversity-logo.svg Source: https://upload.wikimedia.org/wikipedia/commons/9/91/Wikiversity-logo.svg License: CC BY-SA 3.0 Contributors: Snorky (optimized and cleaned up by verdy_p) Original artist: Snorky (optimized and cleaned up by verdy_p)

- File:Wiktionary-logo-v2.svg Source: https://upload.wikimedia.org/wikipedia/commons/0/06/Wiktionary-logo-v2.svg License: CC BYSA 4.0 Contributors: Own work Original artist: Dan Polansky based on work currently attributed to Wikimedia Foundation but originally created by Smurrayinchester

\subsection{Content license}

- Creative Commons Attribution-Share Alike 3.0 